\title{
Scan-Based Volume Animation Driven by Locally Adaptive Articulated Registrations
}

\author{
Taehyun Rhee, Member, IEEE, J.P. Lewis, Member, IEEE, Ulrich Neumann, Member, IEEE, \\ and Krishna Nayak, Member, IEEE
}

\begin{abstract}
This paper describes a complete system to create anatomically accurate example-based volume deformation and animation of articulated body regions, starting from multiple in vivo volume scans of a specific individual. In order to solve the correspondence problem across volume scans, a template volume is registered to each sample. The wide range of pose variations is first approximated by volume blend deformation (VBD), providing proper initialization of the articulated subject in different poses. A novel registration method is presented to efficiently reduce the computation cost while avoiding strong local minima inherent in complex articulated body volume registration. The algorithm highly constrains the degrees of freedom and search space involved in the non-linear optimization, using hierarchical volume structures and locally constrained deformation based on the biharmonic clamped spline. Our registration step establishes a correspondence across scans, allowing a data-driven deformation approach in the volume domain. The results provide an occlusion free person-specific 3D human body model, asymptotically accurate inner tissue deformations, and realistic volume animation of articulated movements driven by standard joint control estimated from the actual skeleton. Our approach also addresses the practical issues arising in using scans from living subjects. The robustness of our algorithms is tested by their applications on the hand, probably the most complex articulated region in the body, and the knee, a frequent subject area for medical imaging due to injuries.
\end{abstract}

Index Terms-Registration, Deformation, Volume Animation.

\section{INTRODUCTION}

\subsection{Motivation}

A $\mathrm{N}$ anatomically accurate 3D human body model including the bones, muscles, tendons, and other anatomical layers is important in various fields. Articulated body regions such as the human knee or hand are capable of a wide range of skeletal movements, resulting in complex deformation of the surrounding soft tissues. Although a physically-based model of important anatomical layers can approximate such deformations, manually creating an accurate physical model including many anatomical layers is difficult and may not exactly mimic the complex interaction and compression among different tissue layers for a specific person.

The difficulty in manually or algorithmically defining complex articulated body structures of an individual subject can be avoided by adopting a data-driven approach. Since scans of a living subject at multiple poses can be used as the training samples, accurate deformable models can be built from actual data. Also, a model constructed from living human scans reflects characteristics of the subject and provides personalized information, which is often essential to create a virtual clone for medical and other applications.

Volume data obtained from 3D medical image scans (e.g. MRI or CT) represents 3D interior anatomy with-

- Taehyun Rhee is with the Samsung Advanced Institute of Technology and U. of Southern California. E-mail: thrhee@samsung.com

- J.P. Lewis is with Weta Digital LTD. and Victoria University.

- Ulrich Neumann and Krishna Nakay are with U. of Southern California.

Manuscript received 17 Dec. 2008; revised 29 May 2009, accepted 3 Oct. 2009 out any occlusion. Translucent volume rendering can successively visualize all these anatomical layers without losing the overall context of the subject. Previous scan based approaches have focused on surface scans and deformation. We develop a data-driven approach in the volume domain using appropriate deformation algorithms, resulting in accurate volume deformation informed by multiple scans of articulated body regions from a living person. Based on our literature survey, we believe this is the first achievement of this type.

One of the challenging issues in scan-based deformation is to obtain geometric correspondences across the volume samples [1]. In case of medical image volumes, the geometrical information is represented by voxel properties without explicit geometric parameterizations, and creating iso-surfaces of each layer from in vivo MRI volumes is very difficult due to poor delineation of different tissue layers.

Our approach uses the only available voxel intensity information without any fiducial markers. To accomplish this, the non-rigid volume registration requires complex non-linear optimization, and the total degrees of freedom (DOF) in the deformation must be carefully controlled. In addition, the optimization must start from proper initialization to avoid the strong local minima that arise in matching articulated subjects.

There are also issues involving the use of in vivo MRI that do not arise with cadavers or non-articulated subjects; these concerns are described in section 2 . 

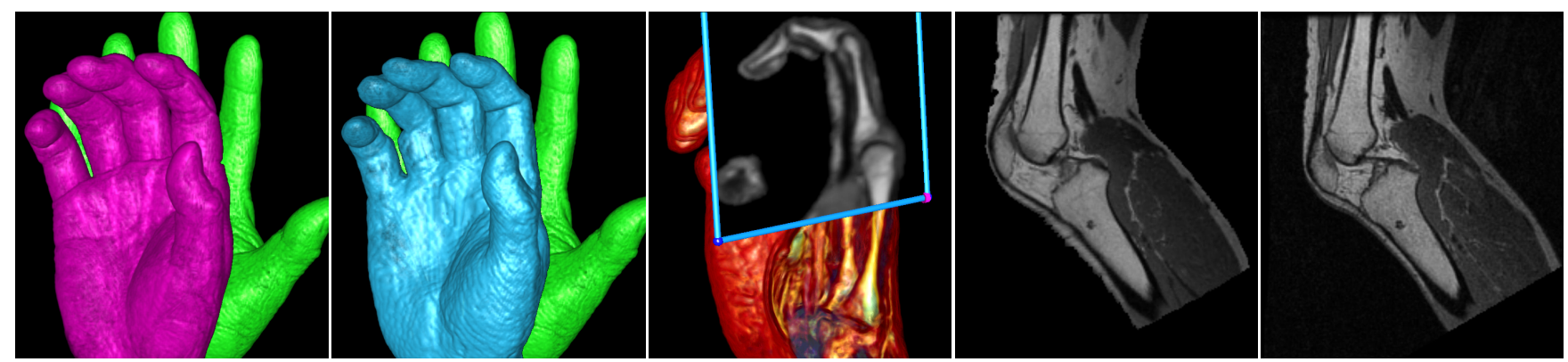

Fig. 1. From the left: human hand volumes obtained from 3D MRI scans (the template volume is green, and the posed scan is magenta), the template volume registered (blue) to the posed scan, volume visualization of the template deformed to an arbitrary pose using example based volume deformation (EVD) (the clipping plane shows the deformed interior), an EVD result for a knee volume, and a ground truth volume which is not included in the EVD training set.

\subsection{Related Work}

\subsubsection{Articulated Human Body Deformation from Scans}

Surface reconstruction methods for 3D human body shapes have been a recent research focus. In [2], [3] a template human body model created from 3D range scans is fit to an individual human body shape guided by sparse 3D markers. Allen et al. [1] demonstrated example-based skin deformation from range scan data and their efforts are enhanced by [4] as a data-driven method for creating deformable human body shapes of individual people. Park et al. [5] capture subtle skin deformation using motion capture devices and around 350 markers. The methods produce very detail animation for subtle skin deformation such as bending, bulging, jiggling, and stretching. The surveyed methods are based on surface scans and they have difficultly capturing inner anatomical layers as well as occluded surfaces (e.g. the palmar skin in a fist pose).

Several volume based approaches have started from the Visible Human data [6], a set of high resolution images captured from a cadaver. Teran et al. [7] generated a physically based articulated body animation of muscular and skeletal body using segmented surfaces of the visible human. Although it is based on volumetric data, the method focuses on the boundary deformations of specific tissues. Volumetric animation of the visible human data has been shown in [8], [9] but their parametric deformation methods could not produce anatomically accurate tissue deformation of complex human tissues. Although the visible human data is high quality, it cannot provide person-specific scans nor multiple pose samples. In [10], an accurate example-based deformable human hand model is derived from multiple CT scans of a living person. Although they capture multiple volumetric scans, they just use skin surface meshes extracted from the volumes. Therefore, the method could not provide adequate solutions for volume deformation. Rhee et al. [11] extended the linear blending skinning algorithm to articulated volume deformation. However the result is constrained by the limitations of the linear blending algorithm and the accuracy of deformation within the soft-tissue volume is not guaranteed.

Physically inspired deformation and other skin defor- mation algorithms should be also surveyed as important areas. However, we entrust the review of these subjects to the papers [12], [7], [13], [14], [15].

Previous efforts for freeform volume deformation also should be noted. Kurzion and Yagel [16] introduces methods to simulate deformation using ray distortion. Recently, this idea has been enhanced to the real-time volume animation system using GPU computation by Westermann and Rezk-Salama [17], [18]. Although this is not the main focus of this paper, we are also fully benefited by recent real-time volume graphics technologies to visualize the result [19].

Large amounts of previous works related to sampled object representation are well surveyed in [20]. Based on this survey of recent related works, articulated human volume animation and inner tissue deformation especially from multiple living human scans of complex skeletal regions (e.g. human hand) appear to be unaddressed issues in the previous research. We present a complete system to solve this problem.

\subsubsection{Non-rigid volume registration}

One of the main issues for scan based volume deformation is to obtain volumetric correspondences across the samples. Approaches using non-rigid volume registration can provide adequate solutions. The literature on image registration and non-rigid deformation is extensive and is surveyed elsewhere [21], [22], [23], [24].

The non-rigid volume registration problem can be solved using non-linear optimization. Therefore, managing the large DOFs of a global deformation function is a central concern to reduce registration cost. The use of methods such as B-splines [25] that are based on regular grids of control points results in unnecessary DOFs.

Adaptive registration has been studied to reduce DOFs and computation times. Rohde et al. [26] used the gradient of global mutual information (MI) to find mismatching regions, with $\mathrm{Wu}$ 's radial basis functions (RBF) [27] providing smooth volume deformation over irregularly allocated sparse control points. Locally measured normalized MI was used by [28] to identify highly mismatched regions requiring additional control points, and thin-plate spline (TPS) radial basis interpolation was 


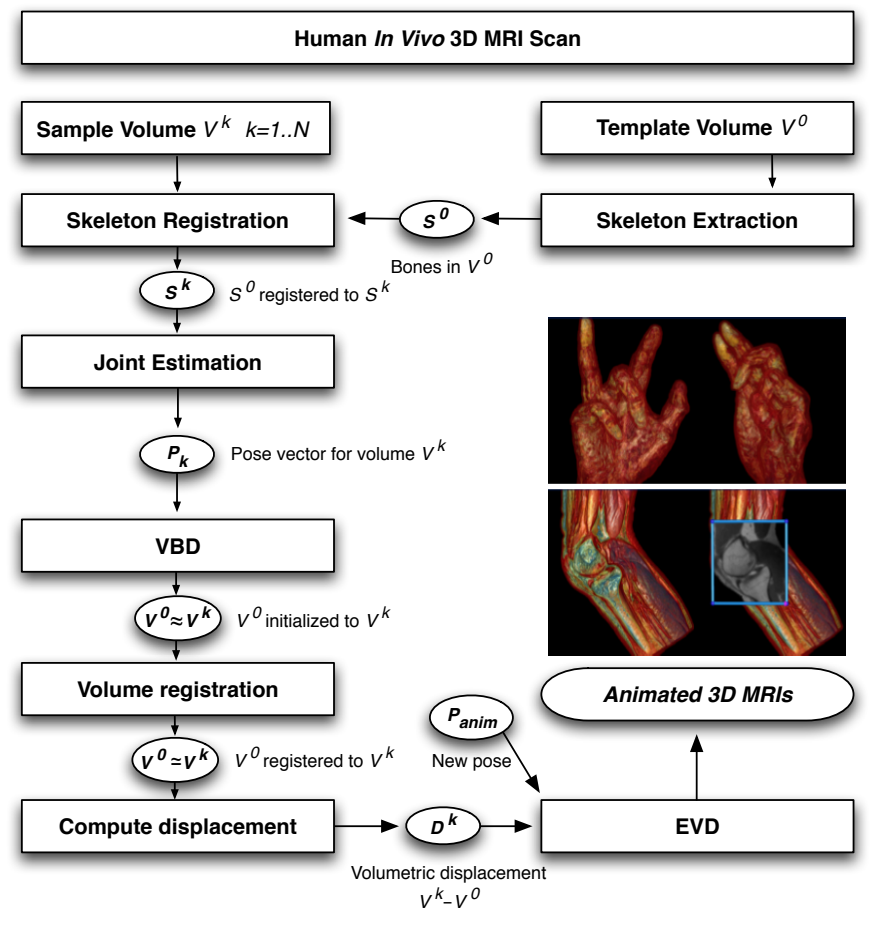

Fig. 2. A complete system to create an anatomically accurate deformable human volume model from multiple scans of a live human subject.

used as a global deformation function.

Previous non-rigid registration studies have been generally focused on non-articulated subjects such as the brain. Although there is much work in rigid articulated registration as reviewed by [21], [29], articulated nonrigid registration has only recently become a focus in medical imaging research. A rigid body registration method for serial lower-limb mouse CT images is presented by [30]. Li et al. [31] demonstrated articulated whole body registration of serial micro CT mouse images. Aische et al. [32] registered neck images using articulated rigid registration and deformation propagation to whole volume though elastic mesh and MI based optical flow.

In recent work by [33], human knee MRI volumes in highly different poses are adaptively registered. The volumetric linear blending algorithm [11] was used for initialization in combination with successive adaptive registration using global deformation functions such as RBFs based on TPS [28].

However, approaches based on global deformation have difficulty registering complex articulated subjects since the computation time of global nonlinear optimization is in general exponential in the number of DOF [34]. We use a novel registration algorithm based on a locally constrained deformation function, thereby reducing the total DOFs and search space efficiently and avoiding the deep local minima that arise in matching complex articulated subjects such as the human hand (e.g. the index finger in one scan aligning to a different finger in subsequent scan).

\subsection{Overview, Contribution, and Applications}

In this paper, we present a complete system to create an anatomically accurate deformable human volume model from multiple living human scans. The results provide an occlusion free person-specific 3D human body model, asymptotically accurate inner tissue deformations, and realistic volume animations of articulated movements, while handling the practical limitations arising in obtaining volume data from articulated living subjects. The overview of the system is shown in figure 2

We approaches the problem in stages. In order to solve the correspondence problem across scans, a particular volume scan is designated as a template and this template is registered to each of the other scans. The use of a template results in a linear number of template-tosample registrations rather than the quadratic number of registrations if scans were registered pairwise. It also produces a correspondence between the template and the scans, thus inducing a common low-level parameterization. The wide range of pose variations is first approximated by volume blend deformation (VBD) [11], providing proper initialization of an articulated subject in different poses.

The initialized template volume is then registered to each volume scan while minimizing a similarity measure such as the mutual information (MI) or sum of squared intensity difference (SSD) without relying on any artificial markers. We present a novel algorithm to efficiently reduce the registration cost while avoiding strong local minima. The algorithm highly constrains the DOFs and search space involved in the non-linear optimization by using hierarchical volume structures and a locally constrained deformation function, the biharmonic clamped plate spline [35].

Compared with previous algorithms using global deformation [33], fewer than one percent of the total DOFs are required (e.g. the total DOFs can be reduced from 7000 to 24). It should be noted that the human hand MRI volume could not be registered using a global registration approach [33] due to strong local minima and dramatically increasing computation time with additional control points; our registration tests with this method failed after running for more than 72 hours. To our knowledge, our locally based approach is the first achievement to register entire human hand MRI volumes in different poses (including soft-tissue deformations) within a reasonable time. The details will be covered in section 4 , and section 6 .

Our registration step establishes a correspondence across scans that permits the last step, a data-driven deformation approach that we extend from surface to the volume domain.

Although the computation time in the construction of the model is substantial, manual guidance is limited to several steps in the initial identification of the skeleton. The model can be displayed with existing volumetric rendering systems and animated with standard 
keyframe techniques; sophisticated motion generation steps such as using motion capture data are beyond the scope of this study and are left for future work. Also, since our algorithm adapts to practical issues involved in using living human scans, the final image quality has necessary limitations compared with previous results using high quality CT scans of cadavers.

In addition to these general contributions, a number of specific applications are foreseeable:

- Medical image registration has many important applications [23], [24]. Our registration algorithm help to extend the range of medical image registration study to articulated body regions, supporting quantification and measurements of tissue deformations required in clinical study of patients suffering from joint pain.

- A moveable articulated volume with realistic treatment of internal tissues could be used in medical education, and surgical simulation as a substitute for a widely used static visible human data.

- The visual presentation of multiple scans of a specific individual as a single coherent model can potentially benefit the patient's (and possibly the doctor's) understanding of problems such as knee injuries, arthritis, artificial limbs, and joint rehabilitation.

- Volume MRI scans of the hand holding an object capture the position and compression of skin regions that are in contact with the object but visually occluded. This information is useful in ergonomics and product design.

\section{3D Medical Image Scans of a Living HUMAN BODY}

Visible light scanning of articulated 3D objects such as the human hand cannot capture 3D interior anatomy, and also cannot capture even the skin surface in poses involving visual occlusion, object contact, or self-contact. Computerized tomography (CT) scanning provides high quality 3D imaging with fine spatial resolution and straightforward segmentation [10], [31], but involves exposure to harmful ionizing radiation, and its associated risks.

We chose to use magnetic resonance imaging (MRI), because it is non-invasive and involves no ionizing radiation and little risk. Compared to CT however, the image quality obtained by MRI is highly limited due to lower signal-to-noise ratio (SNR), non-uniform bone signal, and overlapping intensity ranges between different tissues. As a result, thresholding is not sufficient for segmentation of MRI. In addition, MRI scans of articulated human body regions require careful consideration of subject comfort, scan-time, SNR, and potential for motion artifacts. Our algorithms are designed to handle image quality lower than that of previous approaches involving CT and/or cadaver scans.
The number of obtainable volume samples is in practice limited in the case of human subjects. However, as was demonstrated by [10] in the non-volumetric case, useful models can be built with surprisingly few scans. Our method produces a fully articulated hand model using relatively few scans at critical poses; the details are discussed in section 7 .

TABLE 1

MRI scan parameters

\begin{tabular}{|l|l|l|}
\hline Parameters & Hand & Knee \\
\hline Flip Angle & $15^{\circ}$ & $20^{\circ}$ \\
Volume Size $(\mathrm{mm})$ & $200 \times 200 \times 100$ & $320 \times 320 \times 163.2$ \\
Spatial Resolution $(\mathrm{mm})$ & $0.78 \times 0.78 \times 1.0$ & $1.25 \times 1.25 \times 1.2$ \\
Scan Time per Pose & $5 \mathrm{~min}$. & $6 \mathrm{~min}$. \\
\hline
\end{tabular}

3D MRI volumes of the human hand were captured in ten different poses and 3D volumes of the human knee were captured in four different poses from a healthy male volunteer. The relevant imaging parameters are summarized in Table 1. In order to improve patient comfort and minimize motion artifacts, casts for the hand were made using MR-compatible air-dry clay. This material has virtually no moisture, and produces an MR signal that is below the noise level. Background elimination was not required.

\section{Articulated Volume Initialization}

Although MRI volumes contain the actual skeletal structure, the kinematic joint locations are unknown and must be extracted from the raw volume data. The discovered joint structures for each volume scan provide a compact description of the pose that is used in subsequent articulated volume initialization and for data driven volume deformation.

\subsection{Bone Registration for Pose Estimation}

The actual skeletal structure can be segmented from every raw volume scan, but segmenting all the bones from every volume sample would require unnecessary time and effort. Instead, the bones extracted from a single neutral-pose template volume $V^{0}$ are registered to every other sample $V^{k}$ using intensity based rigid volume registration, and the bone transformation obtained from the registration defines the pose of each sample. When discussing the sub-problem of registering the template to a particular scan $V^{k}$, we will refer to the template as the source volume $V^{s}$ and the particular scan as the target $V^{t}$.

Since automatic bone segmentation from MRI images is difficult [36], [37], those bones related to articulated joint movement are segmented from a template volume using the graph cut algorithm [38] and they are then registered to each volume sample using semi-automatic rigid bone registration [11], [37] as described below.

The oriented bounding box (OBB) of each bone of the template volume is created from the principal axes of 

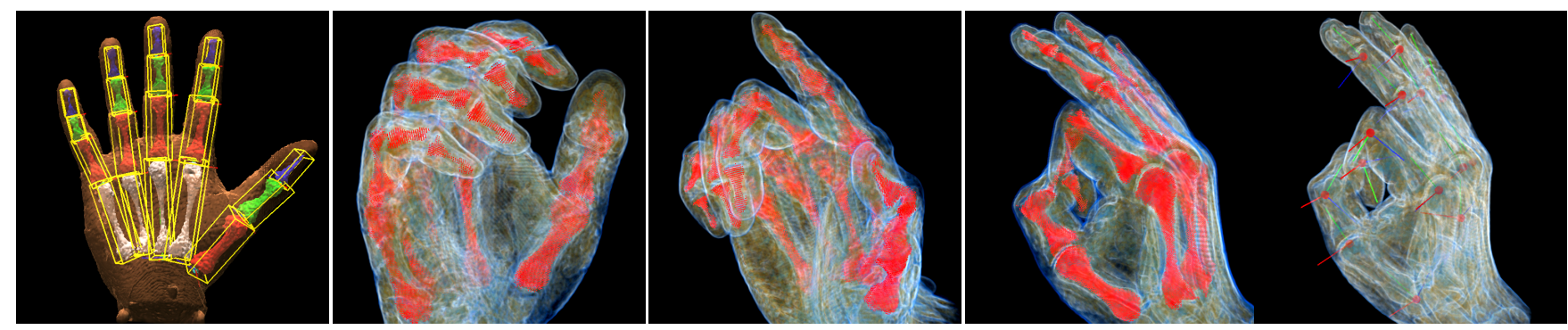

Fig. 3. Bone registration across poses: the left most image is the base volume including extracted bones and their OBBs (yellow boxes); the base bones are then registered (red points) to the other poses; the right most image shows estimated CORs.

the covariance matrix of the bone voxels, and the center of the OBB's base is taken as the origin of the bone's coordinates. In order to support better user control and speed registration [11], the hierarchical kinematic joint structures of the template volume are thus represented by the bone OBB coordinates. The proximal phalanx, middle phalanx, and distal phalanx of each finger are successively linked, and the tibia and fibula are attached to the femur bone, allowing forward kinematics for easy user control.

The bone registration process is semi-automatic. A bone volume is first roughly initialized to the target volume pose using manual transformation of bone OBB coordinates. This bone is then accurately registered to the target volume using intensity based rigid volume registration minimizing the SSD between the volumes,

$$
\min _{T_{j}} \sum_{i=1}^{n}\left|I_{s}\left(T_{j} v_{i}\right)-I_{t}\left(v_{i}\right)\right|^{2}
$$

where $I_{t}$ is the voxel intensity of target bone $j, v_{i} \in V^{s}$ is a voxel grid point of the bone volume in world coordinates, $T_{j}$ is the world coordinate transformation of bone volume $j$ from a source frame to target volume, $I_{s}\left(T_{j} v_{i}\right)$ is the intensity of the source volume at location $T_{j} v_{i}$ obtained with trilinear interpolation, and $n$ is resolution of the bone volume. Although Mutual Information (MI) would be better for multi-modal images, we have a single modality and SSD is adequate for rigid volume registration.

The resulting matrices $T_{j}$ are the world coordinate transformations of each bone volume from the template pose to the corresponding target pose. The concatenated set of all $T_{j}$ registered to target pose $k$ is defined as the pose vector $P_{k}$ that defines the pose of the object during later processing.

From the multiple pose vectors obtained from the bone registration, the center of rotation (COR) of each joint is estimated by the methods presented by [10], [11]. The estimated joints provide intuitive joint control for articulated animation. The results are shown in Fig. 3.

\subsection{Volume Blend Deformation}

The linear blending algorithm [39] was extended to the volume domain by [11] and we call it volume blend deformation (VBD) in this paper. VBD deformation can be written

$$
v_{a}=\left(\sum_{j=1}^{n_{j}} w_{j} T_{j}\right) v_{s}
$$

where $n_{j}$ is the number of joints, $v_{s} \in V^{s}$ is a voxel grid point in the volume $V^{s}$ containing the source pose, $v_{a}$ is a deformed voxel grid point in an arbitrary pose $a$, $T_{j}$ is a homogeneous $4 \times 4$ transformation matrix that transfers joint $j$ from the source pose to the arbitrary pose in world coordinates, and $w_{j}$ is a joint weight that defines the contribution of joint $j$ 's transformations to the deformation. The volumetric weight map to define $w_{j}$ is computed as described in [11]. The detail of the linear blending algorithm is explained in the references [39], [40].

We apply this method for articulated volume initialization to approximately register the template volume to all volume samples $k$. The pose vector $P_{k}$ computed in section 3.1 contains the necessary transformation matrix $T_{j}^{k}$ for use in equation (2). Since the VBD algorithm smoothly deforms the soft-tissue volume while preserving rigid bone regions, a template volume is smoothly deformed to target sample volume $k$ while maintaining the already registered voxels in the rigid bone area. The results of this step are shown in figure 4 .
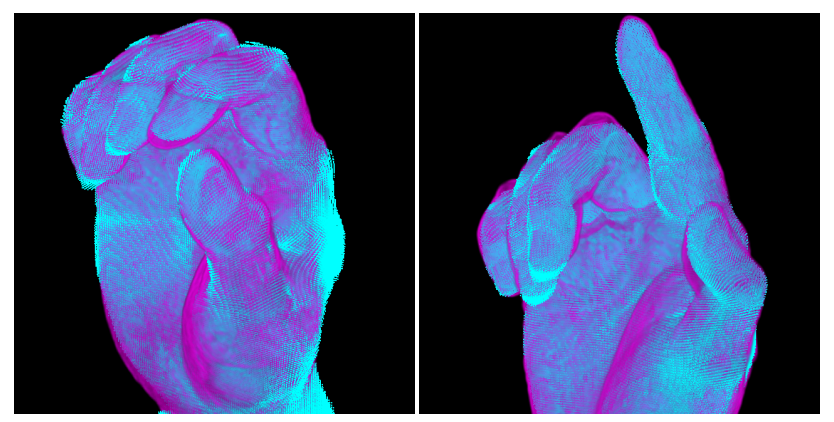

Fig. 4. Articulated volume initialization examples: a template volume (cyan) is initialized to target volume (purple) samples using the VBD algorithm controlled by joint transforms estimated from the target volume. 


\section{Locally Adaptive Non-Rigid Volume REgISTRATION}

Following articulated volume initialization we need to define a non-rigid registration approach capable of accurate and detailed matching while operating on extensive volume data. General non-rigid volume registration methods can be defined by a cost function minimizing the mismatch between the source and target volumes [33],

$$
\max _{\mathbf{f}} S M\left(V^{s}+D\left(V^{s}, \mathbf{c}, \mathbf{f}\right), V^{t}\right)
$$

where $S M$ is a similarity measure such as SSD or MI (in case of SSD the value should be minimized), $V^{s}$ is a source volume, $V^{t}$ is a target volume, $D$ is a warping function to determine the needed additional deformation of volume $V^{s}, \mathrm{c}$ is a control point vector assigned at the target volume frame, $\mathbf{f}$ is the displacement value (warping energy) of the related control point (c, $\mathbf{f}$ are discussed further in section 4.3). In our case, $V^{s}$ is the template scan that will be registered to each of the other (target) scans $V^{t}$. Typically, the cost function is optimized by iterative non-linear optimization and the computation time is determined by both the DOF of deformation function $D$ and the volume size involved in the fit.

We present a method to efficiently reduce the DOF and volume size involved in the optimization while avoiding strong local minima arising from the articulated subject registration. Multi-resolution source and target volumes are created, and the volumes are hierarchically divided into multiple source and target blocks $B_{x, y, z}^{s}$ and $B_{x, y, z}^{t}$ where $x, y, z \in\left\{1 . . n_{x}, 1 . . n_{y}, 1 . . n_{z}\right\}$ and the block resolution $r_{x, y, z}=$ volume resolution ${ }_{x, y, z} / n_{x, y, z}$. The number of blocks $n_{x, y, z}$ is determined by the block hierarchy level $b$; the coarse level $b_{\min }$ has $n_{x, y, z}=8$ blocks.

Locally sufficient (but globally sparse) control points c are adaptively allocated at the block $B_{x, y, z}^{s}$ that has minimum local similarity (maximum mismatch), as described in section 4.3. Then the corresponding local blocks $B_{x, y, z}^{s}$ and $B_{x, y, z}^{t}$ are registered, optimizing the cost function (equation (3)) using the small number of control points.

Global deformation functions such as RBFs based on TPS [28], [33] cannot provide locally constrained deformation and therefore require an excessive number of control points and DOFs to register complex articulated subject such as the human hand. As shown in Fig. 5, control points allocated on a mismatching local block cannot register the local block using a global deformation function. In order to produce a smooth but locally constrained deformation over these local control points, the biharmonic clamped plate spline (described in section 4.2 ) is used as $D$. Whereas many image registration approaches minimize a functional that includes a data fidelity term and an expensive global smoothness term, in our approach globally smooth deformation is obtained by applying a locally smooth deformation adaptively and cumulatively over a hierarchy of scales.

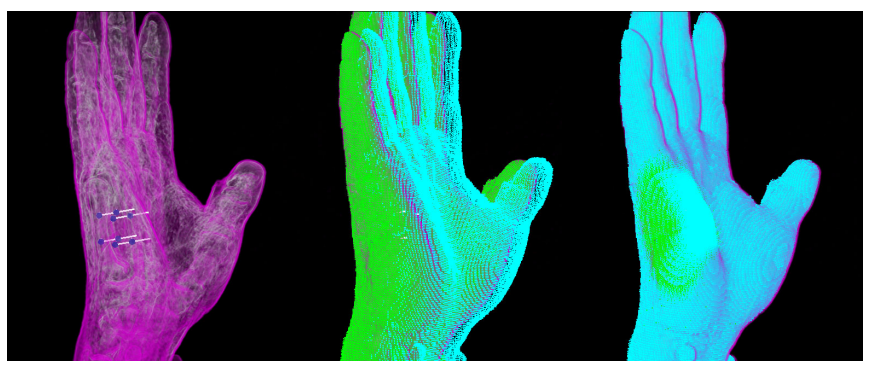

Fig. 5. Global vs local deformation functions: the left image shows allocated control points (blue dots) and the displacements (white bars) to warp a mismatching block; the middle image is the result of a global deformation approach (RBFs based on TPS) warping entire volume; the right image shows the smooth CPS deformation applied to a local block; The source volume is shown in green and the deformed volume is shown in cyan.

\subsection{Volume Similarity Measure}

Similarity measures based on the raw intensity values are attractive because they make use of all the available data and do not require fiducial markers (which are difficult to place inside the body) or prior data reduction and segmentation. For our similarity measure function $S M$, an adaptive combination of SSD and MI is used to measure similarity. In this context SSD is defined as

$$
S M_{S S D}=\sum_{i=1}^{n}\left|I_{s}\left(v_{i}\right)-I_{t}\left(v_{i}\right)\right|^{2}
$$

where $v_{i}$ is a corresponding voxel grid point of source and target volume, and the others are same as equation (1). Since the SSD method is fast and easy to implement, it is widely used for unimodal medical image registration [21]. Also, similarity thresholds defined in terms of intensity are relatively intuitive.

In recent research, MI based similarity measures are widely used for multi-modal image registration, since they can capture any functional relationship between image intensity values. The MI of the image volumes $V^{s}, V^{t}$ is

$$
M I\left(V^{s}, V^{t}\right)=H\left(V^{s}\right)+H\left(V^{t}\right)-H\left(V^{s}, V^{t}\right)
$$

where $H(x)=-\sum P(x) \log P(x)$ and $H(x, y)=$ $-\sum_{x, y} P(x, y) \log P(x, y)$ are respectively the marginal and joint entropies, applied to the histograms of the voxel values. The marginal entropies can usually be considered constant, so maximizing MI entails finding the transformation that minimizes the joint entropy $H(x, y)$ [41], [22]. Pluim et al. [22] surveys a number of variations of this idea. We use a normalized variant of MI by [28], who observed that areas that are relatively featureless are poor candidates for matching. The ratio $M I / \min \left(H\left(V^{s}\right), H\left(V^{t}\right)\right)$ is lowest in areas that have low $M I$ but high detail; these are then the areas that registration should focus on.

For our overall similarity measure function $S M$, MI and SSD are adaptively selected. Since the probability 

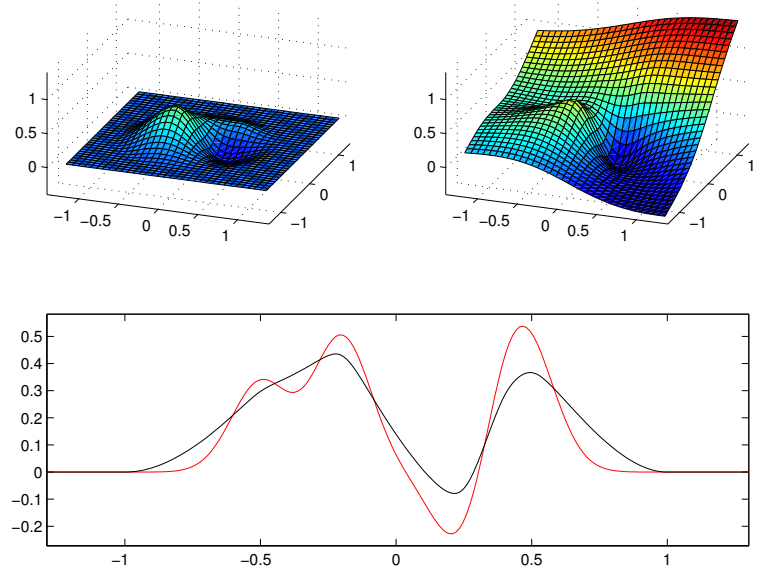

Fig. 6. Top: comparison of the 2D clamped plate spline (left) with a thin plate spline (right) interpolating the same points. The clamped plate spline is both smooth and localized, decaying to zero at the boundary of the unit disc. Bottom: comparison of the 3D biharmonic clamped plate spline (black line) with Gaussian RBF interpolation (red). The plot is the density of a linear section through an interpolated volume. Note that the control points are distributed through the volume and cannot meaningfully be visualized on this plot. The Gaussian RBF interpolation has unnecessary ripples. Increasing $\sigma$ reduces these, but at the cost of increasing the overshoot.

density functions needed by MI can be approximated by histograms only when the histogram contains a sufficient number of values, the MI measure may not be accurate within small regions. We switch from MI at the $\approx 32^{3}$ resolution level to SSD at the $\approx 16^{3}$ level.

\subsection{Biharmonic Clamped Plate Spline}

Because the overall registration is being adaptively split into smaller sub-problems to speed the optimization, we require a deformation that is smooth but localized; see figure 5. Gaussian RBFs would be a default choice for this purpose, but in fact they are not ideal. The Gaussian function is not strictly localized, but this is easily remedied by using a Wendland or Wu basis [42], [27], or by simple thresholding. Another issue is that while Gaussians are smooth in the sense of being infinitely differentiable, they are not smooth in the alternate sense of minimizing a curvature functional $\int(L f(x))^{2} d x$ of a differential operator $L$.

The clamped plate spline (CPS) introduced in [35] is a better solution to the opposing goals of smoothness and locality, as is seen in figure 6. It minimizes the standard spline curvature functional

$$
\int_{\Re^{n}} \sum_{a_{1}+a_{2}+\cdots=m}\left\|\frac{\partial^{m} f}{\partial x_{1}^{a_{1}} \partial x_{2}^{a_{2}} \cdots}\right\|^{2} d \mathbf{x}
$$

which would ordinarily result in a bi- or tri-harmonic solution $\Delta^{2 m} f=0$, but subject to having zero value and derivative on the boundary of the unit disc in $\Re^{n}$. The derivation of the clamped plate spline resembles that of RBFs, with the solution being a weighted sum of Green's function (the operator inverse of $L^{\dagger} L$ ). However, the Green's function in this case is not a radially symmetric function but instead depends on the location relative to the origin. The function for the biharmonic case in three dimensions has been derived $[35]^{1}$,

$$
\left\{\begin{array}{l}
G(x, y)=\|x-y\|(A+1 / A-2) \\
A(x, y)=\frac{\sqrt{\|x\|^{2}\|y\|^{2}-2 x^{T} y+1}}{\|x-y\|}
\end{array}\right.
$$

The $x$ component of the resulting interpolated deformation at a point $\mathbf{p}$ is calculated as

$$
d_{x}=\sum_{k=1}^{n_{\text {feature }}} w_{k} G\left(\mathbf{p}, \mathbf{c}_{k}\right)
$$

(and similarly for the $y, z$ components) where $\mathbf{c}_{k}$ are the locations of the feature points to be interpolated. The weights $w_{k}$ are solved as a linear system

$$
\left[\begin{array}{llll}
G_{11} & G_{12} & G_{13} & \cdots \\
G_{21} & G_{22} & \cdots & \\
\cdots & & & \\
& & &
\end{array}\right]\left[\begin{array}{c}
w_{1} \\
w_{2} \\
\vdots
\end{array}\right]=\left[\begin{array}{c}
d_{1} \\
d_{2} \\
\vdots
\end{array}\right]
$$

where $G_{j k} \doteq G\left(\mathbf{p}_{j}, \mathbf{p}_{k}\right)$ and $d_{k}$ are the desired displacements at the feature locations. Note that the $G_{j k}$ matrix is common to the $x, y, z$ dimensions.

\subsection{Algorithm and Implementation}

$$
\begin{aligned}
& 1 \text { for }\left(b_{\min }<b<b_{\max }\right) \text { do } \\
& 2 \text { compute number of block } n \text { and resolution } r \text {; } \\
& \text { construct block } B_{x y z}^{s}, B_{x y z}^{t} \text {; } \\
& \text { while }\left(\min \left(S M\left(B_{x y z}^{s}, B_{x y z}^{t}\right)\right)<\text { tolerance }\right) \text { do } \\
& B_{\min }^{s, t} \text { is assigned having } \\
& \min \left(S M\left(B_{x y z}^{s}, B_{x y z}^{t}\right)\right) \text {; } \\
& \text { allocate } \mathbf{c} \text { and } \mathbf{f} \text { to } B_{\min }^{s, t} \text {; } \\
& \text { construct } B_{\min }^{s^{*}, t^{*}} \text { using surrounding blocks; } \\
& \text { normalize } \mathbf{c}, \mathbf{f} \text {, and } B_{\min }^{s^{*}, t^{*}} \text { for CPS; } \\
& \text { optimize cost function using } \mathbf{c}, \mathbf{f} \text {, and } B_{\min }^{s^{*}, t^{*}} \text {; } \\
& \text { deform } V^{s} \text { using } D\left(\mathbf{c}, \mathbf{f}^{*}\right) \text {; }
\end{aligned}
$$

Algorithm 1: Registration Algorithm; $b_{\min }$ and $b_{\max }$ are minimum and maximum block level; the other variables are same as defined in the paper; the cost function in line 9 is equation (3).

The volumes are hierarchically divided into small local blocks $B_{x, y, z}^{s}$ and $B_{x, y, z}^{t}$. The pair of corresponding blocks which has minimum similarity value $S M\left(B_{x, y, z}^{s}, B_{x, y, z}^{t}\right)$ can be considered as the most mismatched local region. When such a block $B_{\text {min }}^{s, t}$ is found,

1. Note that Table 1 in [35] has a typographical error: Green's function identified for the triharmonic 3D case is actually the solution for the biharmonic $\left(\nabla^{2}\right)^{2}$ case. 
control points $\mathbf{c}$ are assigned at a small fixed distance inside the corners of the block as in figure 7. As in Park et al. [28], eight control points are used in a $2 \times 2 \times 2$ grid (eight points are sufficient to control the shape of a local volumetric displacement within the block, and the $2 \times 2 \times 2$ placement is easy to visualize and debug). If additional detail is needed it is provided through subsequent refinement levels. This use of an adaptive but deterministic scheme for control point allocation resembles that in quadtree splines [43] and some other adaptive schemes. Note that while irregular placement of the control points could be considered, the additional degrees of freedom would further complicate the optimization problem, and an optimal choice of control point locations is likely to be difficult.

To provide better support for the local registration, the block is enlarged to $B_{\min }^{s^{*}, t^{*}}$ using the surrounding $3 \times 3 \times 3$ blocks. This also provides a boundary region for the CPS to smoothly fade to zero without counteracting the desired effect in the center block. For this purpose,

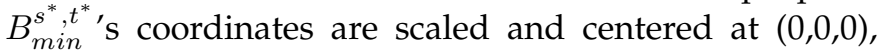
thereby locating all voxel grid points in (-1..1), since CPS requires coordinates within the unit sphere as in figure 7.

Now the minimum similarity source block is registered to the target block while optimizing the cost (equation (3)) for the local block, where $V^{s}=B_{\min }^{s^{*}}$ and $V^{t}=B_{\text {min }}^{t^{*}}$. The result is a set of $x, y, z$ vectors $\mathbf{f}^{*}$ that give the optimal displacement values at the given control points $\mathrm{c}$ for warping the source block to target block. The bone voxels registered in section 3.1 can be used as fixed control points to guide registration and maintain rigidity in the bone region. Since the cost function $S M$ is evaluated over the entire $3 \times 3 \times 3$ region when optimizing the displacement values $\mathbf{f}^{*}$ in the center block, previously computed displacements affecting the neighboring blocks are considered and improved upon.

If the minimum similarity measure of all blocks at level $b$ is less than a given tolerance, the hierarchy level is increased to create additional small blocks to register smaller details. The end result is a progressive registration of the source volume to the target volume while reducing the maximum mismatch across a succession of scales. The progressive registration result is shown in figure 8 and the method is summarized in algorithm 1 and figure 7; one additional loop over the multi-resolution volume is not mentioned in the algorithm summary.

The multi-resolution volume, random restarts of the simplex optimizer, and the locally constrained registration algorithm together efficiently handle the local minima that arise in matching complex articulated subjects.

\section{Example Based Volume Deformation}

The registered volumes now provide correspondence across scans, and the displacements of each voxel grid point between the different poses can be computed. This provides the volume displacements required for

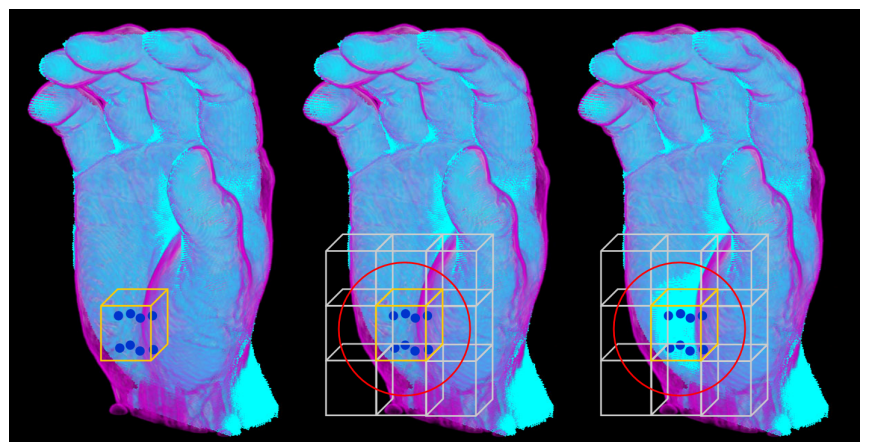

Fig. 7. Locally adaptive non-rigid volume registration: from left, find the maximum mismatching block and allocate control points; construct $B^{s^{*}, t^{*}}$ (front and back blocks are omitted to simplify the figure); register and deform the local block.

data-driven volume deformation, allowing us to manipulate the articulated volume to any arbitrary pose. The example-based volume deformation (EVD) has the general form

$$
v_{a}=E\left(v_{0}+d_{a}\right)
$$

where $v_{0}$ is an undeformed voxel grid point in the neutral-pose template volume $V^{0}, d_{a}$ is a displacement of the voxel grid point in an arbitrary pose $a$, and $E$ is the VBD function in equation 2. The displacement at the arbitrary pose $d_{a}$ is obtained by interpolating the displacements at the training poses $P_{k}$ in a "pose space" [40], [44], [45].

$P_{k}$, the pose vector for volume sample $k$, has been obtained from bone registration in section 3 , and the template volume $V^{0}$ registered to each sample $k$ (section 4) provides corresponding voxel grid geometry $\hat{V}^{k}$. The volumetric displacements between $\hat{V}^{k}$ and $V^{0}$ are calculated by:

$$
d_{k}=\left(\sum_{j=1}^{n_{\text {joint }}} w_{j} T_{j}\right)^{-1} \hat{v}_{k}-v_{0}
$$

where $\hat{v}_{k}$ is a registered voxel grid point in $\hat{V}_{k}$ and $d_{k}$ is the displacement of this voxel grid relative to $v_{0}$. The inverse VBD transfers $\hat{v}_{k}$ to its template pose and the displacement is calculated in the template pose coordinate frame. After defining the displacement of each sample pose, the displacement at an arbitrary pose $d_{a}$ can be smoothly interpolated using RBFs or normalized RBFs [40], [10], [46].

The end result provides the ability to rapidly manipulate the articulated volume to any arbitrary pose while including detailed deformation that interpolates actual data. A posed volume produced by examplebased volume deformation (EVD) is shown in figure 10 and figure 1 . Both the surface and inside anatomical volume layers of the arbitrary pose are adequately reproduced by the EVD algorithm. 


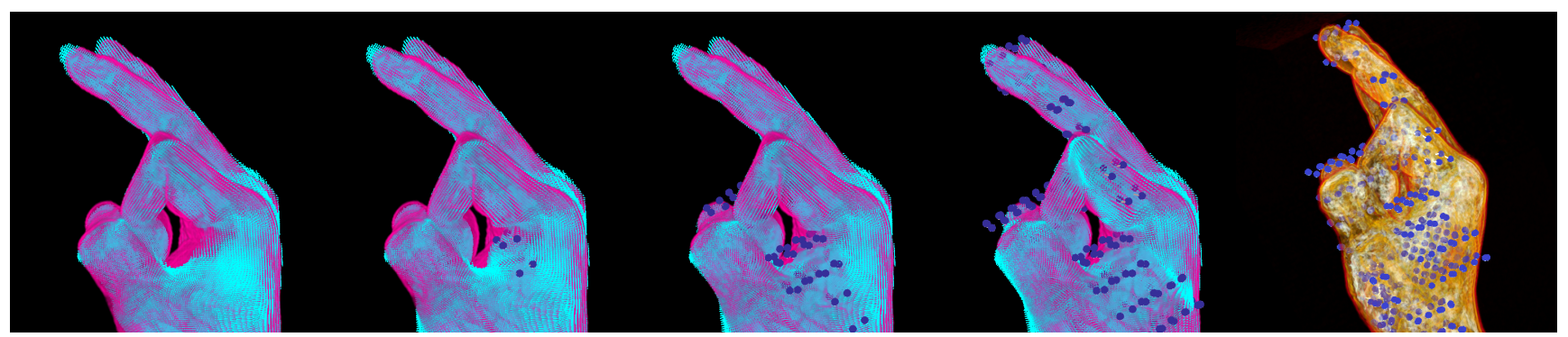

Fig. 8. Progressive non-rigid volume registration results: the template source volume is shown in cyan and the target volume is shown in magenta; The template volume initialized using the VBD algorithm (the left most image) is progressively registered to the target volume using adaptively allocated local control points (block level is 3 ) in the most mismatching region.

\section{Results}

The robustness of our methods is tested by their application on human knee and hand MRI volumes. Four different knee volume poses and ten different hand volume poses were captured while considering practical issues as discussed in section 2 . We approximated the knee joint as one DOF and the hand joint as the 23 DOF which produce the largest articulated motion. The articulated volume initialization in section 3 smoothly manipulates the template volume to different poses. Then, the initialized template volumes are progressively registered to the scan samples using our locally adaptive volume registration algorithm to obtain correspondences across scans.

\subsection{Articulated Volume Registration}

Since no geometric markers are used in the intensitybased registration, validation is usually performed by additional measurements or showing a result obtained with pairs of synthetic data for which the geometric correspondences are known [23], [24]. In this paper, three stages of validations show the robustness of our registration algorithm.

First, we registered synthetically posed knee volumes with small pose variations without articulated initialization. The registration only relies on the locally adaptive registration algorithm in a setting where the ground truth correspondences are known. Voxel grid points identified as inside of the base source volume $V^{0}$ are bent 10 degrees using the VBD algorithm and produce a target volume $\hat{V}^{t} ; 10$ degrees bending generates volume differences that are relatively larger than in typical nonarticulated problems. Using the corresponded geometry between source and target volumes, the Mean Euclidean Distance (MED) between corresponding points can be computed [33]. The tolerance (algorithm 1) for finding minimum similarity block was set to around $1.5 \mathrm{x}$ base MI ( = MI(source (or initialized) volume, target volume), $\approx 18 \mathrm{SSD}$ ) for all the following tests. The MED between source and target is reduced from 5.5 to $3.5(\mathrm{~mm})$ and the Correlation Coefficient (CC) of the two volumes is improved from 0.63 to 0.80 . Table 2 shows the MED, SSD,
CC, and MI voxel similarity measure results. While considering spatial resolution of each voxel $(1.25 \times 1.25 \times 1.2$ $\mathrm{mm}$ ) and volume interpolations for reconstruction, the MED of 3.5 shows accurate results within two voxels of the ground truth result.

\begin{tabular}{|l|l|l|l|l||}
\hline volume & MED & SSD & CC & MI \\
\hline source & $5.5 \mathrm{~mm}$ & 21.3 & 0.63 & 0.52 \\
register & $3.5 \mathrm{~mm}$ & 15.6 & 0.80 & 0.73 \\
\hline
\end{tabular}

TABLE 2

Registration results for synthetic data. MED, SSD, CC, and $\mathrm{MI}$ measures evaluate registration between a template volume and a synthetically posed volume.

Second, following our articulated initialization step, the knee volumes in four significantly different poses are registered. In order to compute the MED between the registered and target volumes, we attached a small number of MR compatible markers on the knee skin surfaces only for the validation purpose; small dots of knee volumes in figure 9 shows the markers. The MED of the markers and other similarity measures with respect to the target volume is shown in table 3 . The registration result of 3.8 MED is reasonable considering the voxel spatial resolution. The results are visually validated in figure 9 .

\begin{tabular}{|l|l|l|l|l||}
\hline volume & MED of markers & SSD & CC & MI \\
\hline source & $18.5 \mathrm{~mm}$ & 41.1 & 0.31 & 0.11 \\
initialize & $5.8 \mathrm{~mm}$ & 18.3 & 0.72 & 0.55 \\
register & $3.8 \mathrm{~mm}$ & 15.7 & 0.79 & 0.65 \\
\hline
\end{tabular}

TABLE 3

Registration results for the knee volume: Mean Euclidean Distance is measured using eight markers; MED, SSD, $\mathrm{CC}$, and $\mathrm{MI}$ are averaged after registering a template pose volume to three other sample volumes.

Finally, we tested our algorithm on a very challenging subject, the human hand (to our knowledge, there are no previous demonstrations of intensity based non-rigid volume registration of the full hand in significantly different poses). In case of the human hand, the complex and varied deformation cannot be meaningfully sampled 

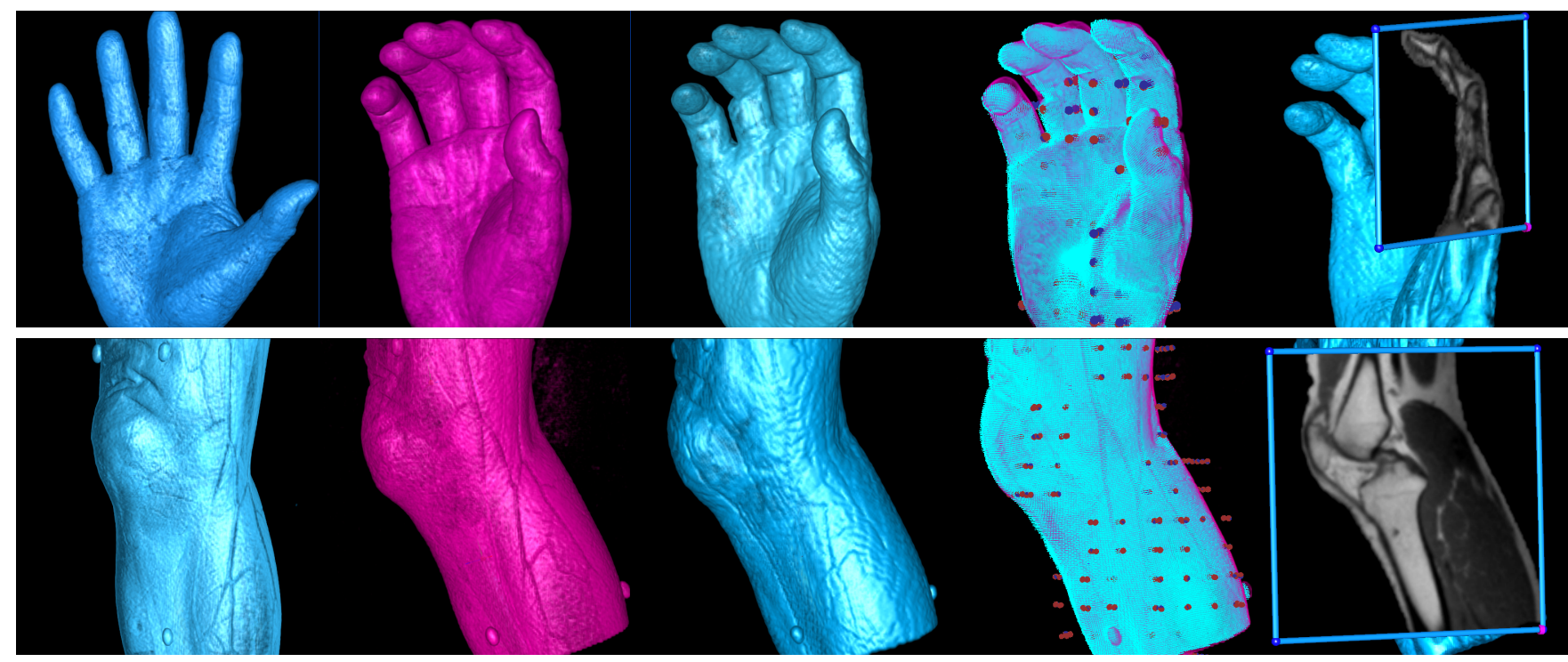

Fig. 9. Non rigid articulated registration; from the left column, template volumes in the neutral pose, target volumes, template volumes registered to the target volumes, differences between registrations (cyan points) and target volumes (magenta) with allocated control points at block level $3\left(32^{3}\right.$ resolution blocks), and interior views of the deformed volume; the markers (small dots) in knee volumes are successfully registered and used only for the validation.

with only a few discrete fiducial markers, so we evaluate the registration using similarity measures and visual validation as was generally accepted in many previous papers [25], [28], [31], [33]. The result are shown in table 4 , figure 8 , and figure 9 .

\begin{tabular}{|l|l|l|l||}
\hline volume & SSD & CC & MI \\
\hline source & 31.6 & 0.01 & 0.07 \\
initialize & 19.4 & 0.53 & 0.23 \\
register & 17.8 & 0.60 & 0.28 \\
\hline
\end{tabular}

TABLE 4

Registration results of the hand volume: SSD, CC, and $\mathrm{Ml}$ are averaged after registering a template pose volume to nine other sample volumes.

Non-rigid volume registration takes a large amount of time [47], [28]. Registration of articulated subjects require vastly more computation time due to the additional DOF, the larger areas of mismatch, and decreased smoothness in the deformation due motion of the skeleton. Since human hand registration requires highly constrained local deformation in the finger areas, registration using global deformation functions [28], [33] did not provide adequate results in our attempts.

Our locally adaptive algorithm adequately registers the articulated knee volumes in 5 hours using around $900 \sim 1250$ total allocated control points at block level 3, and the human hand volumes in an average of 4 hours using $2000 \sim 2500$ allocated control points at block levels $3,4,5$. Although the total DOFs based on the allocated control points are very large (2700 7000), the actual DOFs are always constrained to 24 (eight control points in 3D) at every optimization step; potentially a few more points (less than $4 \times 3$ ) from the bone volume registered in section 3.1 can be considered as fixed control points. The experiments are performed by a machine having a Intel Xeon 3.0GHZ processor with 3 Gbyte memory.

For comparison [28] reported 11-hour compute times for non articulated CT volume registration using 48 fixed control points and a global registration function.

\subsection{Example Based Volume Deformation}

The registered volumes in different poses provide correspondence across samples, allowing volume displacements between the different poses to be computed. The EVD algorithm manipulates a template volume in the neutral pose to any arbitrary pose using kinematic joint control estimated in section 3.

Kurihara et al. [10] obtained an excellent surface deformation model of the hand with only five scans; we use 10 volume scans of the hand for the EVD basis. Recent bio-engineering research showed that most actually encountered human hand poses can be reproduced using a combination of a limited number of principal poses [48]. We tested this idea using 60 different meaningful poses from the "Poser" character system - the 10 dominant eigenposes covered more than $90 \%$ of the variation.

Although the example based volume deformation requires complex pre-computations to handle raw medical volume samples, note that the EVD deformation time itself is small: the time is around 3.5 seconds to deform the human hand volume (255x255x90 voxel grid points) and $1.7 \mathrm{sec}$. to deform the knee volume $(255 \times 255 \times 123$ voxel grid points) to any arbitrary pose. The human hand and knee volume deformed to arbitrary poses are shown in figures 10 and 1 . The volume interior containing all anatomical layers is also adequately deformed and shown via a clipping plane. In figure 11 and 12, 
the VBD, and EVD results are compared with a volume scan excluded from the training set. A real-time volume rendering program [49] is used for display.
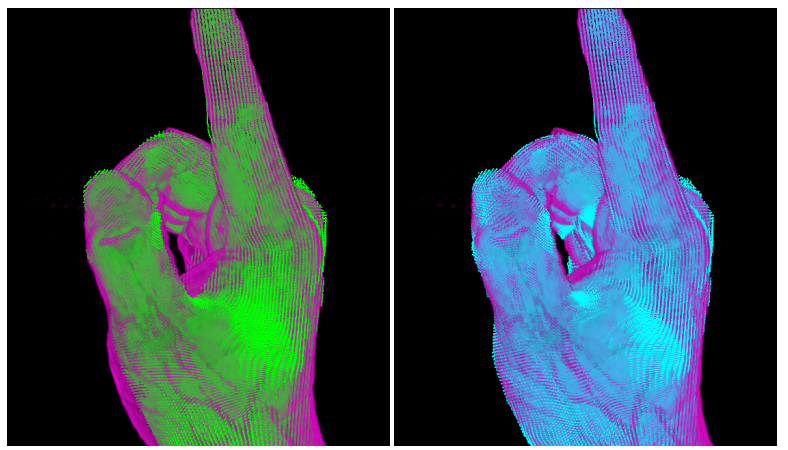

Fig. 11. The VBD and EVD results for the hand. Green (VBD) and cyan (EVD) points represents the deformed volume, and the magenta region shows the difference between the deformation and a ground truth volume

\section{CONCLUSION}

This paper describes a complete system to produce an anatomically accurate scan-based volume deformation model of articulated body regions of a specific individual, while managing the practical limitations arising in multiple volume scans of a living human. In order to provide correspondences across scans, a template volume is registered to each volume sample using VBD initialization in combination with a locally adaptive non-rigid volume registration algorithm that efficiently reduces computation cost while avoiding the strong local minima inherent in articulated body registration. The end result is a person-specific articulated deformable volume model with anatomical accuracy well beyond current state of the art systems that are generally based on a single cadaver scan with simple parametric deformation.

The approach is demonstrated on human knee and hand MRI volumes. Both subjects are challenging and important for many applications. In particular, the human hand is one of the most complex articulated human body regions. It is difficult to scan and therefore produces poor MRI images with unclear tissue boundaries. Based on our survey, our results may be the first to register full human hand MRI volumes in highly different poses using intensity-based non-rigid registration. Given the results obtained with this complex subject, we feel there is a good argument that the method will work better for many simpler cases as shown in our result using knee MRIs. Previous registration papers and skin deformation papers have often shown restricted examples [40], [1], [26], [28], [31], [24].

To our knowledge, fully volumetric scan-based deformation from living human scans has never been attempted in the previous work. A model constructed from living human scans provides personalized information, which is often essential for medical applications.
Therefore, our model can substitute for previous cadaver based static models in many applications while providing the full advantages of manipulatable volume data.

Our contributions are evident and many applications are feasible as described in section 1.3. On the other hand, due to the challenging problems associated with scans from living humans, several limitations were encountered. Our methods for volume registration and data-driven volume deformation are fully automated except for the bone extraction and initialization steps, which are partially automated. Manual guidance in these steps would not be necessary if the CT scans were used, since bones can be reliably segmented with simple thresholding. However, CT scans involve ionizing radiation.

Since we set the MR parameters to cover the entire knee volume, the MR signals on the patella (knee cap) and tendons were not distinguishable. As we skipped the patella during the manual segmentation, the region was not properly initialized for the subsequent non rigid registration, resulting in some artifacts. Custom MRI setting or CT scans can provide better data resulting in easy segmentation and accurate registration. Although we approximated the knee joint as one DOF for simple animation control, more DOF should be considered for accurate control.

An obvious question arises concerning the accuracy of using a limited number of scans in the EVD of the hand. This is not an intrinsic limitation as the system can accommodate more scans. However, as discussed in section 6.2 , the number of scans needed is fewer than one might expect.

Since we focused on several challenging problems in this initial study, some important related issues were skipped and left for future work. More accurate joint estimation, sophisticated motion generation using motion capture, and high quality volume visualization should be considered for further study, but are beyond the scope of this paper.

As we discussed, most of the limitations of our method come from the challenges of using scans of articulated regions of living subjects. Although we can solve many of the addressed limitations using CT scans of cadaver volumes while increasing quality of registration and final animation, results obtained from in vivo subjects have obvious advantages and are highly evaluated in medical and biomechanics research. The trade off between the higher quality visual output from a cadaver (e.g. [6]) or CT scans versus the practical limitations arising from using living human subjects may be a good discussion issue in this study.

\section{ACKNOWLEDGMENTS}

We appreciate the sincere feedback from anonymous reviewers, and thank the many researchers in radiology, biomechanics, medical imaging, and computer vision at our institutions for useful discussions. 

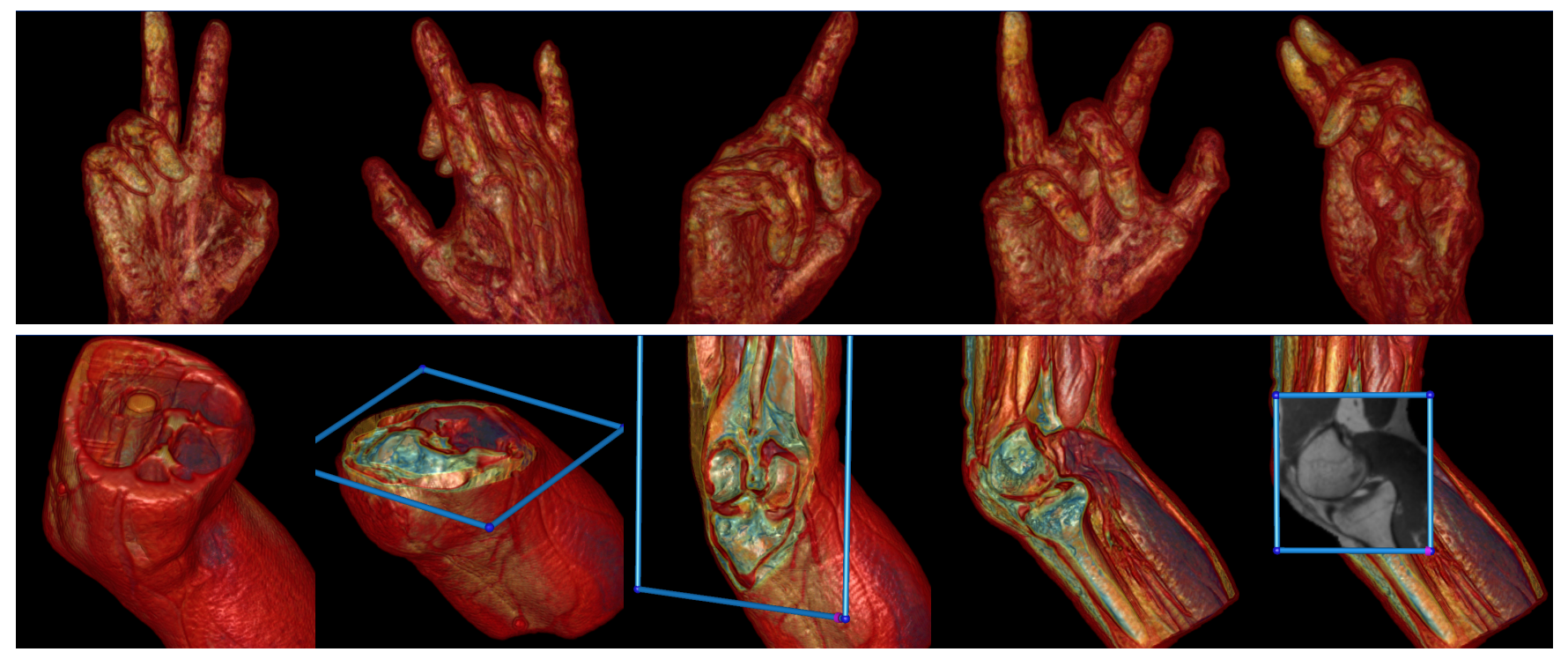

Fig. 10. Volume deformation by EVD: the human hand and knee volume are deformed to arbitrary poses; translucent volume rendering visualizes the complex anatomical layers without losing the context of the subject; a clipping plane shows the interior MRI volumes.
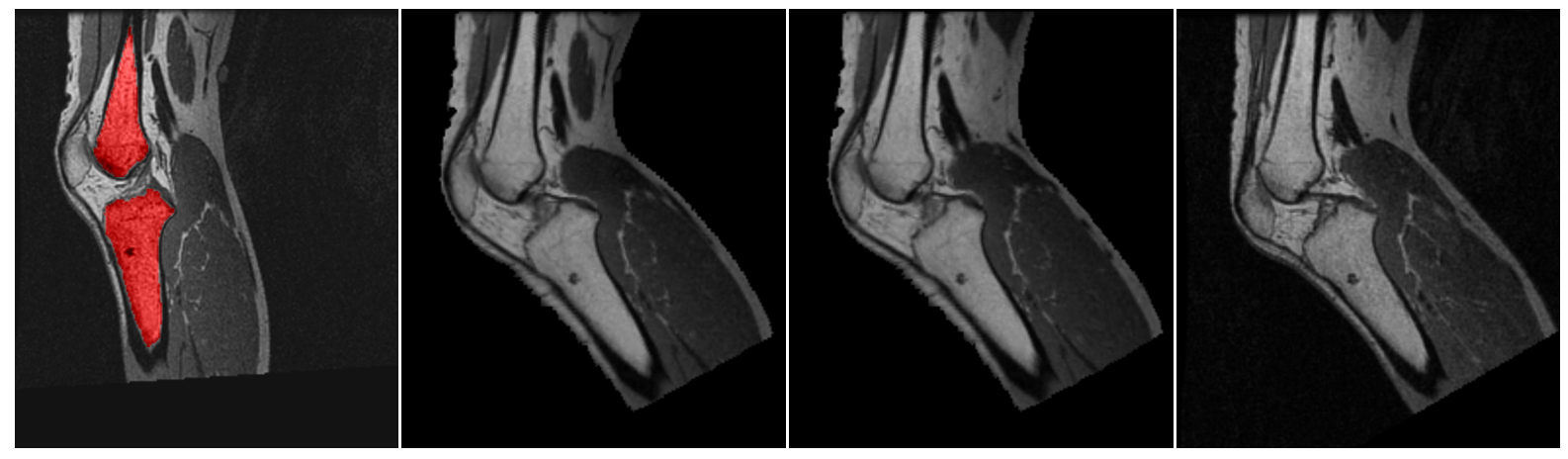

Fig. 12. The VBD and EVD results for the knee. From the left: a template volume in the undeformed neutral pose (the red area shows segmented bone volumes), the VBD and EVD results of the knee volume deformed to ground truth volumes that are not included in the training set, and the ground truth knee volume. Please see the accompanying video for additional examples.

\section{REFERENCES}

[1] B. Allen, B. Curless, and Z. Popović;", "Articulated body deformation from range scan data," in SIGGRAPH '02: Proc. Computer Graphics and Interactive Techniques. ACM Press, 2002, pp. 612-619.

[2] B. Allen, B. Curless, and Z. Popović, "The space of human body shapes: reconstruction and parameterization from range scans," ACM Trans. Graphics (SIGGRAPH '03), vol. 22, no. 3, pp. 587-594, 2003.

[3] H. Seo, F. Cordier, and N. Magnenat-Thalmann, "Synthesizing animatable body models with parameterized shape modifications," in Proc. ACM SIGGRAPH/Eurographics Symposium on Computer Animation (SCA '03), 2003, pp. 120-125.

[4] D. Anguelov, P. Srinivasan, D. Koller, S. Thrun, J. Rodgers, and J. Davis, "Scape: shape completion and animation of people," in ACM Trans. Graphics (SIGGRAPH '05). ACM Press, 2005, pp. 408-416.

[5] S. I. Park and J. K. Hodgins, "Capturing and animating skin deformation in human motion," ACM Trans. Graphics (SIGGRAPH '06), vol. 25, no. 3, pp. 881-889, 2006.

[6] "Visible human project," National Library of Medicine, 1986, http://www.nlm.nih.gov/research/visible.

[7] J. Teran, E. Sifakis, S. S. Blemker, V. Ng-Thow-Hing, C. Lau, and R. Fedkiw, "Creating and simulating skeletal muscle from the visible human data set," IEEE Trans. Visualization and Computer Graphics, vol. 11, no. 3, pp. 317-328, 2005.

[8] N. Gagvani and D. Silver, "Animating volumetric models," Graph. Models, vol. 63, no. 6, pp. 443-458, 2001.

[9] V. Singh and D. Silver, "Interactive volume manipulation with selective rendering for improved visualization," in Proc. IEEE Symposium on Volume Visualization and Graphics (VV '04), 2004, pp. 95-102.

[10] T. Kurihara and N. Miyata, "Modeling deformable human hands from medical images," in Proc. ACM Symposium on Computer Animation (SCA '04), 2004, pp. 357-366.

[11] T. Rhee, J. Lewis, U. Neumann, and K. Nayak, "Soft-tissue deformation for in-vivo volume animaton," in Proc. Pacific Graphics, 2007, pp. 435-438.

[12] I. Albrecht, J. Haber, and H. P. Seidel, "Construction and animation of anatomically based human hand models," in Proc. ACM SIGGRAPH/Eurographics Symposium on Computer Animation (SCA '03), 2003, pp. 98-109.

[13] L. Kavan and J. Zara, "Spherical blend skinning: A real-time deformation of articulated models," in ACM SIGGRAPH Symposium on Interactive 3D Graphics and Games (SI3D '05). ACM Press, April 2005, pp. 9-16.

[14] D. L. James and C. D. Twigg, "Skinning mesh animations," ACM Trans. Graphics (SIGGRAPH '05), vol. 24, no. 3, pp. 399-407, Aug. 2005. 
[15] B. Merry, P. Marais, and J. Gain, "Animation space: A truly linear framework for character animation," ACM Trans. Graphics, vol. 25, no. 4, pp. 1400-1423, 2006.

[16] Y. Kurzion and R. Yagel, "Space deformation using ray deflectors," in Proc. Sixth Eurographics Workshop on Rendering (Rendering Techniques '95). Springer-Verlag, 1995, pp. 21-30.

[17] R. Westermann and C. Rezk-Salama, "Real-time volume deformations," Comput. Graph. Forum, vol. 20, no. 3, 2001.

[18] C. Rezk-Salama, M. Scheuering, G. Soza, and G. Greiner, "Fast volumetric deformation on general purpose hardware," in Proc. ACM SIGGRAPH/EUROGRAPHICS Workshop on Graphics Hardware (HWWS '01). ACM, 2001, pp. 17-24.

[19] K. Engel, M. Hadwiger, J. Kniss, C. Rezk-Salama, and D. Weiskopf, Real-Time Volume Graphics. A. K. Peters, 2006

[20] M. Chen, C. Correa, S. Islam, M. W. Jones, P.-Y. Shen, D. Silver, S. J. Walton, and P. J. Willis, "Manipulating, deforming and animating sampled object representations," Computer Graphics Forum, vol. 26, no. 4, pp. 824-852, 2007.

[21] J. V. Hajnal, D. L. Hill, and D. J. Hawkes, Medical Image Registration. CRC Press, 2001

[22] J. P. W. Pluim, J. B. A. Maintz, and M. A. Viergever, "Mutual information based registration of medical images: A survey," IEEE Trans. Medical Imaging, vol. 22, no. 8, pp. 986-1004, August 2003.

[23] W. R. Crum, T. Hartkens, and D. L. G. Hill, "Non-rigid image registration: theory and practice," The British Journal of Radiology, vol. 77, pp. 141-153, 2004.

[24] M. Holden, "A review of geometric transformations for nonrigid body registration," IEEE Trans. Medical Imaging, vol. 27, no. 1, pp. 111-128, 2008.

[25] D. Rueckert, L. I. Sonoda, C. Hayes, D. L. Hill, M. O. Leach, and D. J. Hawkes, "Nonrigid registration using free-form deformations: application to breast MR images." IEEE Trans. Medical Imaging, vol. 18, no. 8, pp. 712-721, August 1999.

[26] G. K. Rohde, A. Aldroubi, and B. M. Dawant, "The adaptive bases algorithm for intensity-based nonrigid image registration," IEEE Trans. Medical Imaging, vol. 22, pp. 1470-1479, 2003.

[27] $\mathrm{Z}$. Wu, "Solving PDE with radial basis function and the error estimation," in Lecture Notes on Pure and Applied Mathematics, 1998, p. 202.

[28] H. Park, P. H. Bland, K. K. Brock, and C. R. Meyer, “Adaptive registration using local information measures," Medical Image Analysis, vol. 8, no. 4, pp. 465-473, 2004.

[29] G. E. Marai, D. H. Laidlaw, and J. J. Crisco, "Super-resolution registration using tissue-classified distance fields," IEEE Trans. Medical Imaging, vol. 25, no. 2, pp. 177-187, 2006.

[30] X. Papademetris, D. Dione, L. Dobrucki, L. Staib, and A. Sinusas, "Articulated rigid registration for serial lower-limb mouse imaging," in MICCAI '05, 2005, pp. 919-926.

[31] X. Li, T. Peterson, J. Gore, and B. Dawant, "Automatic registration of whole body serial micro CT images with a combination of point-based and intensity-based registration techniques," in IEEE International Symposium on Biomedical Imaging (ISBI '06), April 6-9 2006, pp. 454-457.

[32] A. B. D'Aische, M. D. Craene, B. Macq, and S. Warfield, "An improved articulated registration method for neck images," in IEEE Engineering in Medicine and Biology, Sep 1-4 2005, pp. 76687671.

[33] T. Rhee, J. Lewis, K. Nayak, and U. Neumann, “Adaptive nonrigid registration of 3D knee MRI in different pose spaces," in IEEE International Symposium on Biomedical Imaging (ISBI '08), March 14-17 2008.

[34] S. Boyd and L. Vandenberghe, Convex Optimization. Cambridge, 2004.

[35] S. Marsland and C. J. Twining, "Constructing diffeomorphic representations for the groupwise analysis of nonrigid registrations of medical images," IEEE Trans. Medical Imaging, vol. 23, no. 8, pp. 1006-1020, 2004.

[36] S. Komojima, N. Miyata, and J. OTA, "Identification of position and orientation of hand bones from MR Images by bone model registration," in Proc. IEEE/RSJ Int. Conf. Intelligent Robots and Systems, 2004.

[37] N. Miyata, M. Kouchi, M. Mouchimaru, and T. Kurihara, "Finger joint kinematics from MR Images," in Proc. IEEE/RSJ Int. Conf. Intelligent Robots and Systems, 2005.

[38] C. Rother, V. Kolmogorov, and A. Blake, "GrabCut: interactive foreground extraction using iterated graph cuts," in $A C M$ Trans. Graphics (SIGGRAPH '04). ACM Press, 2004, pp. 309-314.
[39] N. Magnenat-Thalmann, R. Laperrière, and D. Thalmann, “Jointdependent local deformations for hand animation and object grasping," in Proc. Graphics Interface '88, Jun. 1988, pp. 26-33.

[40] J. P. Lewis, M. Cordner, and N. Fong, "Pose space deformation: a unified approach to shape interpolation and skeleton-driven deformation," in SIGGRAPH '00: Proc. Computer Graphics and Interactive Techniques. ACM, 2000, pp. 165-172.

[41] P. Viola and I. W. M. Wells, "Alignment by maximization of mutual information," in Proc. International Conference on Computer Vision (ICCV '95). IEEE Computer Society, 1995, p. 16.

[42] H. Wendland, "Piecewise polynomial, positive definite and compactly supported radial functions of minimal degree," Advances in Computational Mathematics, vol. 4, no. 1, pp. 389-396, 1995.

[43] R. Szeliski, "Motion estimation with quadtree splines," IEEE Trans. Pattern Analysis and Maching Intelligence, vol. 18, no. 12, pp. 1199-1210, 1996.

[44] P.-P. J. Sloan, C. F. Rose, and M. F. Cohen, "Shape by example," in Proc. ACM Symposium on Interactive 3D Graphics (SI3D '01). ACM Press, 2001, pp. 135-143.

[45] P. G. Kry, D. L. James, and D. K. Pai, "EigenSkin: Real time large deformation character skinning in hardware," in Proc. ACM SIGGRAPH Symposium on Computer Animation (SCA-02), 2002, pp. 153-160.

[46] T. Rhee, J. Lewis, and U. Neumann, "Real-time weighted posespace deformation on the GPUs," Computer Graphics Forum (Proc. Eurographics '06), vol. 25, no. 3, pp. 439-448, 2006.

[47] L. Zagorchev and A. Goshtasby, "A comparative study of transformation functions for nonrigid image registration," IEEE Trans. Image Processing, vol. 15, no. 3, pp. 529-538, 2006.

[48] R. H. Clewley, J. M. Guckenheimer, and F. J. Valero-Cuevas, "Estimating effective degrees of freedom in motor systems," IEEE Trans. Biomedical Engineering, vol. 55, no. 2, pp. 430-442, 2008.

[49] J. Kniss, G. Kindlmann, and C. Hansen, "Interactive volume rendering using multi-dimensional transfer functions and direct manipulation widgets," in Proc. IEEE Visualization, 2001, pp. 255262.

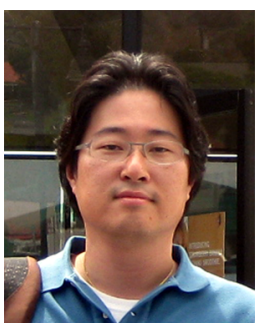

Taehyun Rhee is a Senior Researcher and R\&D Staff Member of 3D Graphics \& VR Group at Samsung Advanced Institute of Technology (SAIT) in Samsung Electronics Co., LTD. He received his Ph.D. in Computer Science from University of Southern California in 2008 and M.S. in Computer Science and Engineering from Sogang University in 1996. His research concern is to solve scientific problems related to Computer Graphics, Computer Animation, Computer Vision, and Medical Image. His current research activity is focused on realistic human body modeling and animation, soft-tissue deformation, surface/volume reconstruction from in vivo scans, medical image, and general purpose GPU computation for realtime applications. He was a Senior Engineer of Research Innovation Center at Samsung Electronics from 1996 to 2003. He developed photorealistic rendering algorithms, rendering system, 3D user interfaces, VR applications, and consulted 3D industrial design process.

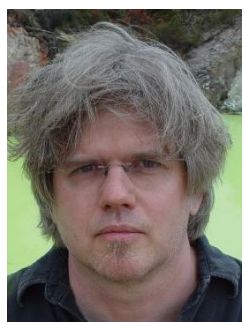

J.P. Lewis is a research programmer at Weta Digital and Senior Lecturer at Victoria University in New Zealand. In the past he has worked as a Research Associate at the University of Southern California and Stanford University, and in the film industry at Industrial Light and Magic, Disney, and elsewhere. Lewis has published more than 40 papers on a variety of topics and has several patents and film credits. $\mathrm{He}$ is a member of the IEEE Computer Society. 


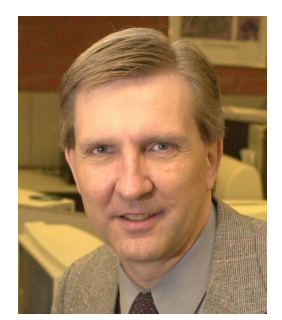

Ulrich Neumann is a Professor of Computer Science, with a joint appointment in Electrical Engineering, at the University of Southern California. He directs the Computer Graphics and Immersive Technologies (CGIT) Laboratory and is the Associate Director for Research at the NSF's Integrated Media Systems Center (IMSC). He completed an MSEE from SUNY at Buffalo and a computer science Ph.D. from the University of North Carolina at Chapel Hill, where his focus was on parallel algorithms for interactive volume-visualization. His current research relates to augmented virtual environments, 3D modeling, and visualization. He won an NSF CAREER award in 1995 and the Jr. Faculty Research award at USC in 1999. Prof. Neumann held the Charles Lee Powell Chair of Computer Science and Electrical Engineering while the Director of the Integrated Media Systems Center (IMSC), an NSF Engineering Research Center (ERC) from 2000-2004. He has published over one hundred peer-reviewed articles and serves on numerous journal and conference committees, including serving as Associate Editor for the IEEE Transactions on Multimedia. In his commercial career, he designed multiprocessor graphics and signal processing systems, cofounded a video game corporation, and developed and licensed electronic products.

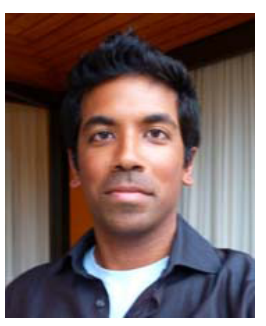

Krishna Nayak is an Associate Professor of Electrical Engineering at the University of Southern California, with courtesy appointments in Biomedical Engineering, Radiology, and Medicine. He received his PhD in Electrical Engineering from Stanford University in 2001. His research focus is magnetic resonance imaging, with an emphasis on pulse sequence design and image reconstruction. His current projects include dynamic cardiac and body imaging, steady-state free precession imaging, myocardial perfusion imaging, body composition assessment, dynamic upper airway imaging, and applications of compressed sensing. 